\title{
Deployment of Open Source ERPs: What Knowledge Does It Require?
}

\author{
Björn Johansson and Vadim Koroliov \\ Department of Informatics, School of Economics and Management, Lund University, \\ Ole Römers väg 6, \\ SE-223 63 Lund, Sweden \\ bjorn.johanssondics.lu.se, v.koroliovegmail.com
}

\begin{abstract}
Enterprise resource planning (ERP) systems are rapidly becoming a de facto standard in business activity. While large and medium-sized companies have the luxury to afford proprietary ERP solutions, small companies are struggling with resource poverty which maybe makes them consider available open source ERP products which are free from licensing fee. However, there is little knowledge available on open source ERP adoption in small companies. In order to spread some light on the first phase of ERP adoption, an experiment on open source ERP deployment was conducted. The experiment aimed at investigating what knowledge is required to successfully deploy open source ERP systems. The experiment was based on a research framework, the Technology Acceptance Model 2 (TAM2), and considered usability testing and user training and education factors. The factors of Perceived Ease of Use (PEOU) and Perceived Usefulness (PU) were used to determine the ease of deployment process, and the usefulness of the open source ERP deployed in relation to the made effort. The findings suggest that user with advanced computer skills perceive open source ERP deployment process as easy, and the deployed open source ERP was seen as being useful to organizations business activities.
\end{abstract}

Keywords: Deployment experiment, Enterprise resource planning systems, ERPs., Open source, Small organizations, SMEs.

\section{Introduction}

In organizations, there is a growing need for managing information to become competitive and sustain such advantage over a longer period of time. Therefore, many organizations have implemented extensive enterprise information systems, such as enterprise resource planning (ERP) systems, to obtain a better control over information and thereby acquire advantages over its competitors [1] by, for instance, getting better and faster access to information stored. However, deployment can be problematic for organizations since it can take long time and cost a lot of money; and there is also a high risk that it may fail [1-3]. Despite that, there is an increased interest in ERPs among organizations which also has created an interest in developing 
simplified versions of ERPs. These simplified versions are developed both by proprietary ERP vendors, such as Microsoft Dynamics or SAP, as well as by new vendor organizations in the open source (OS) area. The open source ERP projects could be seen as an alternative to traditional ERP proprietary systems available today. In general it can be suggested that OS have grown large and still continues to grow strongly as more and more organizations become interested in how they can benefit from OS in their organization [4]. Therefore, it could be suggested that organizations, in their search for having an ERP system implemented in their organization, could consider an open source ERP system. Consequently, an interesting question follows if this is doable or not. From an earlier investigation done by Johansson and Sudzina [5], we know that there are a lot of open source ERPs available for download from an online service such as SourceForge. On the other side, what we do not know is, if this is done or if it is feasible for an organization to do so? This directs to the question discussed in this paper which is: What knowledge is required to successfully deploy open source ERP systems?

To be able to say something about this, Section 2 defines open source ERP and open source ERP deployment. Section 3 presents the research conducted, which could be briefly described as a controlled experiment of downloading and making a first usage test of an open source ERP system. The experiment aims at describing knowledge needed to successfully deploy open source ERP systems. Section 4 analyzes the results from the experiment and discusses the knowledge required to successfully deploy open source ERP systems. In the final section some conclusions are provided, and some future research questions in the context of open source ERP deployment are suggested.

\section{ERPs, Open Source ERPs and Open Source ERP Deployment}

Enterprise Resource Planning systems (ERPs) are major investments for organizations, and according to Morabito et al., [6] have high attention among practitioners, academia, and media. They state that ERP research primarily focuses on two aspects: 1) organizational and economic impact of ERP implementation, and 2) how to best manage implementation. There are a number of key characteristics that more or less all ERP system share making them a unique subtype of information systems. Firstly, ERP is defined as a standardized packaged software [7] designed to integrate entire organization [8-10], its business processes and ICT into a synchronized suite of procedures, applications and metrics which transcend organizational boundaries [11] and that can be bought (or rented) from an external provider and adapted to firm's specific requirements.

The fact that ERPs are assumed to integrate the organization (both interorganizationally as well as intra-organizationally) and its business process into one package, feeds the complexity of ERPs when it comes to development and implementation as well as usage [12]. Millman [13] posits that ERPs are the most expensive but least-value-derived implementation of information and communication technology (ICT) support. The reason for this, according to Millman, is that a lot of ERPs functionality is either not used or is implemented in a wrong way. In addition, wrong implementation results from ERPs being customized to fit the business 
processes, instead of changing the processes to fit the ERP [13], described by Hammer and Champy [14] as "paving the cow path".

Several studies on inspiring success [15], but also failures [16, 17], associated with implementation and utilization of ERPs [18] exist. Benefits are only related in part to the technology, and most come from organizational changes such as new business processes, organizational structure, work procedures, integration of administrative and operative activities, and global standardization of work practices leading to organizational improvements, supported by the technology [19]. It can definitely be said that implementation of ERP systems is a difficult and costly organizational "experiment" [18], and implementation of ERP systems can be described [20] as "perhaps the world's largest experiment in business change" and for most organizations "the largest change project in cost and time that they have undertaken in their history". The implementation is a necessary but insufficient prerequisite for benefits and value, at least for having competitive parity [21].

According to Wieder et al. [22] there is no significant performance difference between ERP adopters and non-adopters either on process or overall firm levels. In conclusion, it can be claimed that there are different opinions on benefits and advantages of ERP systems adoption.

Nevertheless, the market tendency shows that ERP adoption is growing and will grow among organizations worldwide. Jacobson et al. [23] denote - in their report on ERP market sizing - that ERP investments among large corporations as well as small and medium-sized enterprises (SME) are continuously increasing. The ERP systems became in practice an industry standard [24] and, as argued by Shehab et al., [25] considered to be the price of entry for running a business.

From this it can be said that the ERP products market have grown. Up to recent years, the ERP systems offer has been primarily characterized by proprietary software products. The largest ERP vendors to present are SAP, Oracle, Infor and Microsoft. In the same time, the industry witnessed the proliferation of open source ERP packages as well. The open source ERP packages, and namely community-based versions, provide a free alternative to commercial ERP packages. Some notable examples are OpenBravo, Compiere, TinyERP, OFBiz, Adempiere, xTuple/PostBook and others, and the majority of them target SMEs.

One would wonder why they are free. Usually, the companies who stand behind these products earn money in a different way, other than traditional. Along with the community version, which is completely free, there are commercial versions with better support, updates and upgrades.

In brief, open source ERP vendors bring a couple of reasons of why to choose open source ERP. Firstly, there are no upfront licensing fees. Anyone can download the software from vendor's website and try the product for free. Thus, assess whether it suits or not the company's needs. Secondly, open source offers free control of software customization with the support of contributing communities and organizations. There is also professional-quality support available from companies working with that specific ERP. And one of the last arguments is that open source reduces the specification risk, characteristic to custom built software; and loss of vendor risk (Opentaps.org, 2011). Not less important is the promise of "be up and running with a full system in 10 minutes" (xTuple.com, 2011) - this, in particular, makes it very interesting to challenge. 
Fougatsaro [26] presents the following seven reasons of why organizations should choose open source ERP:

- Flexibility - the available source code makes it easier to customize and integrate the open source ERP with existing systems.

- Quality - as a result of commitment of vendors and communities to development efforts.

- Ability to adapt to business environment

- No hidden costs - as opposed to proprietary ERP systems, where changes scalability issues might be imminent.

- Ability of specific developments - freedom of customizing and development

- Free of vendor dependence - the support is provided both by community and commercial vendors.

- Freedom to upgrade or not.

Fougatsaro [26] claims that despite all these benefits, open source software also has disadvantages. The comparison of proprietary and open-source (community/free) ERP advantages and disadvantages, as well as a total cost of ownership analysis have been researched to a limited extent. As stated by Carvalho [27] the full picture of open source ERP's issues and benefits still has to be covered. Therefore, it might be difficult to evaluate which type of ERP brings the most value. However, this of course impacts the decision when adopting or not, but this is outside the scope of this paper that focuses on open source ERP deployment and knowledge required for doing that.

\subsection{Open Source ERP Deployment in SMEs}

In recent years, ERP systems have become very attractive to SMEs, i.e. the SMEs' interest towards ERP system has increased. And this happened due to a number of reasons. Firstly, ERP vendors have shifted their development efforts focus from mainly large customers - today a saturated market, to small and medium sized companies - a promising market both in cash and in customers. Consequently, the range of ERP packages offer has considerably increased, adjusting to the needs and pockets of various companies [28]. Secondly, it is the highly dynamic business environment which requires ERP adoption in order to gain competitive advantage over rivals [28]. In the same context, Jacobson et al., [23] mention that ERP adoption among SMEs comes as a response to new customer requirements, as well as to the wish to participate in a highly global market. And finally, ERP systems offer unprecedented advantages over any other traditional models of managing businesses [29].

However, before any further statements are made about SMEs and ERPs, it is important to define SMEs. In this context SMEs are defined as: companies from 10 to 49 employees are considered to be small, companies from 50 to 249 employees are considered being midsized, and companies having 250+ employees are considered to be large companies. This definition is consistent with how the European Commission [30] defines SMEs. However, this paper regards organizations with less than 10 employees (micro) as small, the reasons are that constraints and objectives of ERP systems in this group of organizations could be considered the same [31]. However, 
as Laukannen et al. state, there are significant differences between small and mediumsized companies and therefore they should not be considered as a homogeneous category. The same is claimed by Carvalho and Johansson [32].

In terms of general constraints, small companies, in comparison to medium-sized, have a lower user IT competence and insufficient information, but are less sensitive to changes enforced by ERP implementation. In terms of ERP objectives, medium-sized companies feel eager to develop new strategic ways of doing business, and are more interested in expanding its business activity [31].

It can also be said that small companies, despite having a growing interest for ERP, have a low actual rate of ERP adoption. Laukannen et al. [31] point out that resource poverty is one the main constraints of ERP system adoption, i.e. they cannot afford one. Small companies, in comparison to mid-size and large enterprises, have limited or scarce resources. Then the logical question arises: so why not chose the free ERP?

Somewhat apparent, the financial factor can be crucial and decisive for small companies. However, Johansson and Sudzina [5] mention that cost is not the only factor affecting the selection and adoption processes of an open source ERP. Laukannen et al. [31] suggest that knowledge is another determinant barrier lying in the way of ERP adoption in small companies specifically, whether it is IT competency, enough information for decision-making in ERP selection or system usage.

It is crucial to understand that knowledge requirements change along various stages of ERP life cycle and embrace a large set of skills, experiences, abilities and perspectives [33]. In other words, type of knowledge required in implementation phase, for example, would be different from what knowledge is needed in the system use phase. However, there is no scientific support whatsoever for knowledge requirements for open source ERP deployment.

Given that a small company intends to adopt an open source ERP for free, it would be both interesting and challenging to find out how much knowledge is needed or how easy it is to deploy - choose, implement and use - an open source ERP, and when deployed if it meets the expected outcomes/benefits.

\section{The Open Source ERP Deployment Experiment}

The objective of the open source ERP deployment experiment was two-folded. On one hand, it was to find out the required knowledge in the open source ERP adoption process in small companies, and namely, the actual prerequisites, needs and issues with an open source ERP in the early stage of adoption, i.e. its deployment. The prerequisites, needs and issues are to cover the time and knowledge aspects. On the other hand, it is also interesting if the open source ERP is found useful in relation to the effort required to deploy it.

The data collected was based on TAM2 model developed by Venkatesh and Davis [34]. In other words, TAM2 model was used as lens for assessing the open source ERP deployment process (Perceived Ease of Use) and its primary impact on business and its users (Perceived Usefulness). The experiment focused on three questions: 
- How much effort - time and knowledge - does it take to deploy an open source ERP?

- What is the perceived ease of use in the deployment stage?

- What is the perceived usefulness of open source ERP in the deployment stage?

\subsection{The Foundation of the Experiment}

In the realities of small companies, where the resources and knowledge are limited, open source ERPs seem to be a viable solution. First of all, the product is free regarding license cost, so initial costs could be said are low. However, the problem of knowledge still remains, because it is still unclear how much knowledge is required to adopt and use an open source ERP. Therefore, it would be interesting to find out how much effort is required to deploy and start using open source ERP systems. In order to address this issue, the Technology Acceptance Model 2 was used.

\subsection{Technology Acceptance Model 2 (TAM2)}

TAM2 comes as an extension to the initial Technology Acceptance Model (TAM) developed by Davis [35]. Both of the models or both versions address the subject of system usage. The model helps to understand and evaluate the reasons and factors which affect the use and adoption of new systems or existing systems. Also, the TAM model is viewed as a tool to measure users will and intention to adopt a system. In this research papers, TAM2 will also serve as a measuring tool to assess the ease and usefulness of an open source ERP.

The initial model was limited to two factors, which in opinion of its author, affect the intention and use of a system: perceived ease of use (PEOU) and perceived usefulness (PU). Despite its popularity and use, Venkatesh and Davis [34] suggested an extension. The extended features take into consideration the external factors which affect perceived usefulness (see Figure 1). Venkatesh and Davis [34] explain that external factors have social and cognitive character.

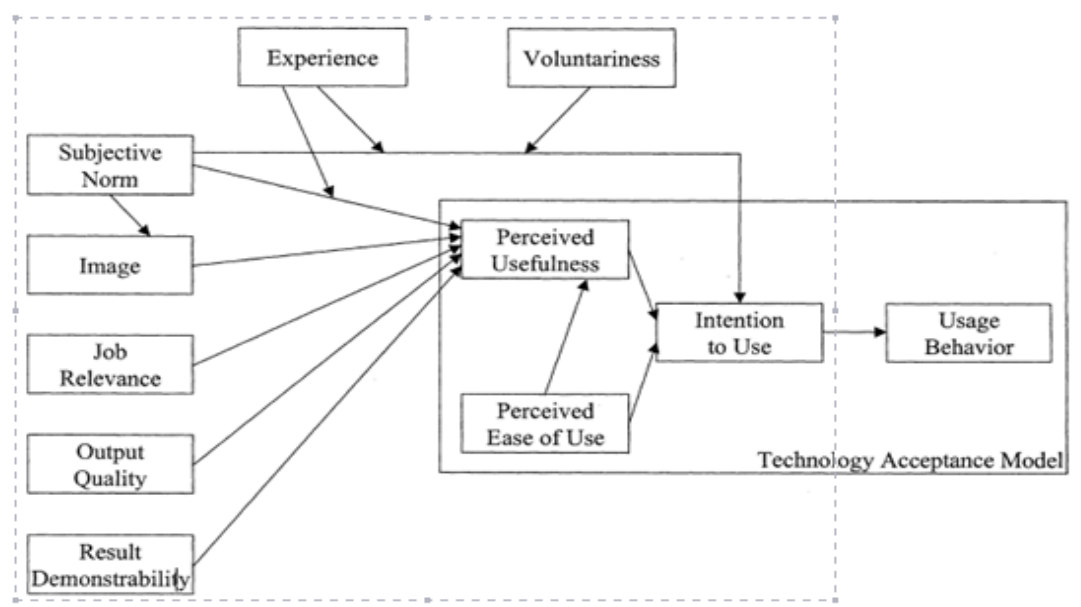

Fig. 1. The TAM2 model and scope of this research [34] 
Perceived usefulness is the extent the user thinks the systems helps her or him to perform the tasks, i.e. job activities. Perceived ease of use measures the effort required to use a system, i.e. how easy it is to perform the job tasks. PEOU affects the PU and the Intention to Use. One user's intention to use affects in turn the actual usage of the systems, i.e. determines the Usage Behavior.

The external factors are explained by Venkatesh and Davis [34] as follows: Subjective norm, is a "person's perception that most people who are important to him think he should or should not perform the behavior in question". The key idea behind is that persons are susceptible to other people' ideas and views. That is why some persons perform actions motivated by other people.

Voluntariness is "the extent to which potential adopters perceive the adoption decision to be non-mandatory" [34]. In other words, voluntariness determines if system use is perceived as obligatory and unwilling or opposite to these.

Image has a positive effect on perceived usefulness, if the use of the system is to enhance user's social status or image; otherwise it has a negative effect on perceived usefulness. Experience might also have a positive or negative effect on PU. It is considered that a system is most like to be perceived useful if the user is experienced.

Job relevance, as defined by Venkatesh and Davis [34], is "an individual's perception regarding the degree to which the target system is applicable to his or her job". In other words, job relevance measures whether a system use is important to daily job tasks.

Output Quality measures the quality level of the tasks performed by the system. In other words, if the task performed by the system is perfect, then the perceived usefulness of the system is appreciated higher.

And ultimately, Result Demonstrability measures the tangibility of the results given by a system. Venkatesh and Davison [34] explain that "even effective systems can fail to garner user acceptance if people have difficulty attributing gains in their job performance, specifically to their use of the system". That is why it is important for users to see direct results of their system use, in order for the system to be perceived as useful.

This research paper's scope is limited to the deployment of a system, i.e. the installation and first use of an open source ERP system. That is why the use of the TAM2 model would be partial (the dotted line in Figure 1). And namely, the interest lies on perceived ease of use and perceived usefulness of the open source ERP in its installation and first use.

Consequently, the paper will focus on defining the effort needed for and usefulness of deploying an open source ERP in the realities of a small company, in terms of time and knowledge.

\subsection{The Pre-study of Open Source ERP Deployment Experiment}

The pre-study started with finding information about available open source ERPs on WWW. Having taken the suggestion of Johansson and Sudzina [5], the first source to look for open source software was www.sourceforge.org, which is the largest project promoting open source software. Using this portal's search function, the keywords "open source ERP" were queried. Over 13,000 hits came up, all of these were obviously not relevant. 
According to sourceforge.org, the two most popular and downloaded open source CRM \& ERP solutions were OpenBravo and PostBooks. So the next logical step was to check out the website of respective projects, and the information provided.

Both of the websites made a professional impression. OpenBravo impressed by the number of customers, and both of the open source ERPs have a large community of users. With no previous experience related to ERP and its activity, the deciding criteria for choosing one was the technical criteria and requirements. It was relatively easy to find the download page and proceed to downloading. Both software products offered solution for cloud, Linux and Windows platforms, however PostBooks/xTuple offered also for Apple/MAC OS users.

Having consulted the available documentation, first difficulties appeared. OpenBravo promised a very simple, one-click installation for Linux users, but more complicated installation paths for other platforms, i.e. advanced computer skills are needed. xTuple software promised easy installation on all platforms. Having available only Linux and Windows operating systems, it was decided to try out OpenBravo on Linux and xTuple on Windows XP. Indeed, the installation process was very easy for OpenBravo, but with no transparency whatsoever. Only, advanced computer users could be able to follow the installation procedure. After installing, OpenBravo on Linux/Ubuntu the software ran in web browser successfully. Regarding, xTuple the installation procedure was as easy, plus the level of procedure transparency and clearness was quite high. The user is guided by explanatory instructions with options of choosing elements to be installed. By default, all elements are being installed. The software also ran with no errors on Windows XP. The approximate time for both installations was ten to fifteen minutes, with high speed internet available. At this point, it is important to mention that from the technical standpoint, the requirements are not high. The computer properties available were 1GB RAM, 10GB free space on hard drive, and Windows XP. The basic needs for installation are mentioned on the vendors' websites.

It was decided to proceed with xTuple. The argument behind was the availability and spread of Windows operation systems, so that theoretically most small companies would have a computer running Windows. The next step was to get acknowledged with xTuple open source ERP.

The log-in procedure was very easy. However, potential users have to pay attention to details while installing, because important information is given, such as credentials, which would be of use later on.

After log-in, the first thing was to get accustomed with menus available in the software. xTuples offers a very pleasant user interface, with large buttons and well organized modules, such as sales, inventory and other. For screenshots please visit www.xtuple.com.

After getting to know the software, the decision was to consult available documentation and tutorials on how to work with the ERP. There are many videos available; some of the videos are introductory, and some of them give detailed instructions on the internals and functions of the system. From the video tutorials, it was found out that following steps were required in order to get a valid invoice ${ }^{1}$ :

1 The following experiment aimed at having students downloading, installing and print an invoice with data from a business case. 
1. Register a new user for the company

a. Create separate account

b. Enter details such as address, company info, logo etc.

2. Register the new customer with according details

3. Create a new product/item

4. Configure the taxation settings

5. Create a new sales order containing the product created

6. Ship and print out the invoice.

These steps were enough to print an invoice valid for Swedish standards, i.e. having required information. From the knowledge gained so far the actual deployment experiment took place.

\subsection{The Study of Open Source ERP Deployment}

There were two methods used for data collection, a structured questionnaire; that gathered data on students' general information profile, as well as on computer experience and knowledge. The purposes of questionnaire was to establish whether experiment participants had any previous experience which resembled the experience required in accomplishing the task, i.e. installing and using software.

The other data collection method was semi-structured interviews, giving the freedom to express the issues and thoughts regarding the easiness of deploying an open source ERP. The interviews were audio recorded, and after completion immediately transcribed.

The experiment involved three students, who were asked to download an open source ERP system; and then proceed with installing it. In the next phase, the students were asked to print out an invoice. In order to simulate a real business situation, a business case with enough data to print the first customer invoice was provided to them. And for the case to resemble a real business situation, all data contained were enough to make a valid invoice by means of Swedish regulations, i.e. VAT, Address details, Organizational number etc. During the experiment, the participants were continuously asked to assess the level of knowledge and effort required to complete a certain task. Printing of invoice was not set as the ultimate goal, but rather as a guiding objective which would take an amount of effort and user interaction with the software. Of great interest was the whole process, from its start to its end - printing the invoice. It is important to mention and understand, that this experiment shares features with observational studies and usability tests. Firstly, the intention of the experiment is to answer to the research questions posed by observing its participants in the settings close to reality, i.e. in front of the computer in an office. Lastly, the experiment shares the features of a usability test, where the system is tested for ease of use. However, the focus of this study is not the interface nor the productivity, but rather the whole experience of the participants from the very moment of deciding to look for an open source system to the very last moment of printing the first invoice.

The sampling technique was influenced by the organizational characteristics of small companies. According to Laukannen et al. [31], size is the most significant 
factor which shapes the attitude of organizations towards ERPs. The attitude is expressed in terms of constraints and objectives for ERP adoption. In their study, a small enterprise was characterized by an average of 29 employees, with low IT competence, resource poverty, and representing a variety of industries: wholesale, logistics, retail and manufacturing. This tells that there are no strict requirements on choosing participants, as long as types of companies studied are various, and there are no strict requirements for IT skills. However, in order to avoid bias or misinterpretation accurate profiling of participants computer skills have been made.

The questions in the interview were constructed around two important aspects: 1) Perceived Ease of Use and 2) Perceived Usefulness.

The profiling questions, as previously mentioned, had the purpose of establishing the background of participants and their level of computer skills.

The questions related to Perceived Ease of Use had the purpose to assess the level of ease perceived in the open source ERP deployment process. As TAM2 is a quantifiable model, the participants were asked to answer PEOU questions on a scale from 1 to 5 , were 1 was ranked as very easy and 5 - very difficult. Also, clarifying questions followed in order to get better explanation of why participants gave specific grades. The questions related to Perceived Usefulness were designed to assess the positive or negative influence of TAM2 external factors on PU.

The TAM2 model has been applied in two ways. The interviewees were first asked to grade the Perceived Ease of Use related to deployment experiment stages on a Likert-scale. This was done in order to assess the effort and knowledge needed to evaluate the process for the amount of effort needed to complete the tasks. After that, the interviewees were asked to elaborate on the effect of external factors on Perceived Usefulness, whether it was negative or positive. That was done in order to determine if the open source ERP delivered the benefits expected.

\section{Analysis of the Deployment Experiment}

In this section a summary of the empirical results will be presented. The structure follows the theoretical model and research questions, and, thus, the data will be arranged accordingly into the following parts: Profiling, Perceived Ease of Use, Perceived Usefulness. Perceived Ease of Use will cover subtopics: Finding and Choosing an open source ERP, Installing and Configuring an open source ERP, Accomplishing the Business Case tasks.

The interviewees who took part in the deployment experiment are all master students within Information Systems field. The computer experience has been assessed as between intermediate and advanced levels, with computer experience being seven years on average. Having been asked on their use of computers, they commonly replied that internet and studies are the main reasons. Only one of the students used computer for work and multimedia also. All students confirmed that they have successfully installed software on computers. Regarding software usage, all students have been using one or more software products for a long time, five years or more; and all of them have advanced skill level with the product they have been using over that period of time. 
All students were given a business case, used as a guide in the deployment experiment. In brief, the experiment participants were asked to choose, install, configure and accomplish the task of printing the first invoice. These stages have been assessed for knowledge and time effort.

\subsection{Finding and Choosing an Open Source ERP}

Having been asked to find and choose an open source ERP, all students searched for one on Internet. The most common search keywords used were "open source ERP", "open source ERP free" and "open source ERP download". Asked to explain the logic behind the search actions, students clarified that they were willing to evaluate the search results given by Google according to their relevance. In other words, the students chose the first results on the Google's webpage, after having queried for open source ERP. The resulting webpage included for instance OpenBravo, xTuple, Compiere, Opentaps.

To the question on how easy it was to find an open source ERP, they all stated that finding one was very easy.

The students proceeded with looking at the websites available in the search results. Asked how the students evaluated different OS ERP choices, they gave different answers. One student took into consideration the professionalism of the website. In other words, he considered the looks and the design of the website. The rest of participants did not have any explanation for the choice. Ultimately, the users selected to proceed with xTuples/PostBooks open source ERP.

However, it is crucial to mention that none of the students took into consideration the technical parameters and requirements of the open source ERP.

\subsection{Installing and Configuring an Open Source ERP}

All students showed the same behavior during installation process. None of them gave too much consideration to installation and configuring information. They all proceeded with preconfigured elements.

One of the students explained that this behavior is due to lack of knowledge about certain parts of software, such as databases offered and other elements; adding that "sometimes this is scary just because there are things configured you don't know anything about it". But in order to be on the safe side, the students chose to install all elements suggested. Also, the rush can be explained by the wish to run and try out the software at once, skipping the configuration and installation details.

When asked to share the first impression about the installation and configuration process, they expressed that the process was very easy and took little time and effort.

\subsection{Getting the First Invoice}

This part of assignment was the most challenging and most complex for the students. Some of the issues happened right in the beginning of the task, when they could not find the credentials needed to login to the enterprise resource system. However, all three found the interface of the software very "handy" and pleasant. 
Having familiarized themselves with the interface and menus, they proceeded with the task. During the task, they all stated that the software was intuitive and helped them in achieving their goal. However, it also created partial confusion due to multiple reasons such as lack of knowledge, lack of supporting help, and no process transparency from the software. The last reason specifically is related to the save function of the software which was not notifying about its results, such that creating confusion whether the data was saved or not.

Before accomplishing their task, only one of the participants decided to turn to available help on Internet, video tutorials and ERP documentation on the vendor's website. It is interesting to mention that the same respondent used the same technique, googling, for solving issues whenever a problem appeared. The other two used their intuition and the menus available in the software.

Ultimately, the experiment participants succeeded to print out the first invoice. It is crucial to mention that the resulted invoices lacked all data required in the business case.

\subsection{Perceived Ease of Use}

Generally speaking, students graded the deployment process as relatively easy. The average grade, on a scale of one (very easy) to five (very difficult), was two. The most difficulties were faced when configuring and working with the ERP in order to type in necessary data and print out the invoice. Finding, downloading and installing the open source ERP was described as a very easy task. However, more transparency in the process was seen as necessary.

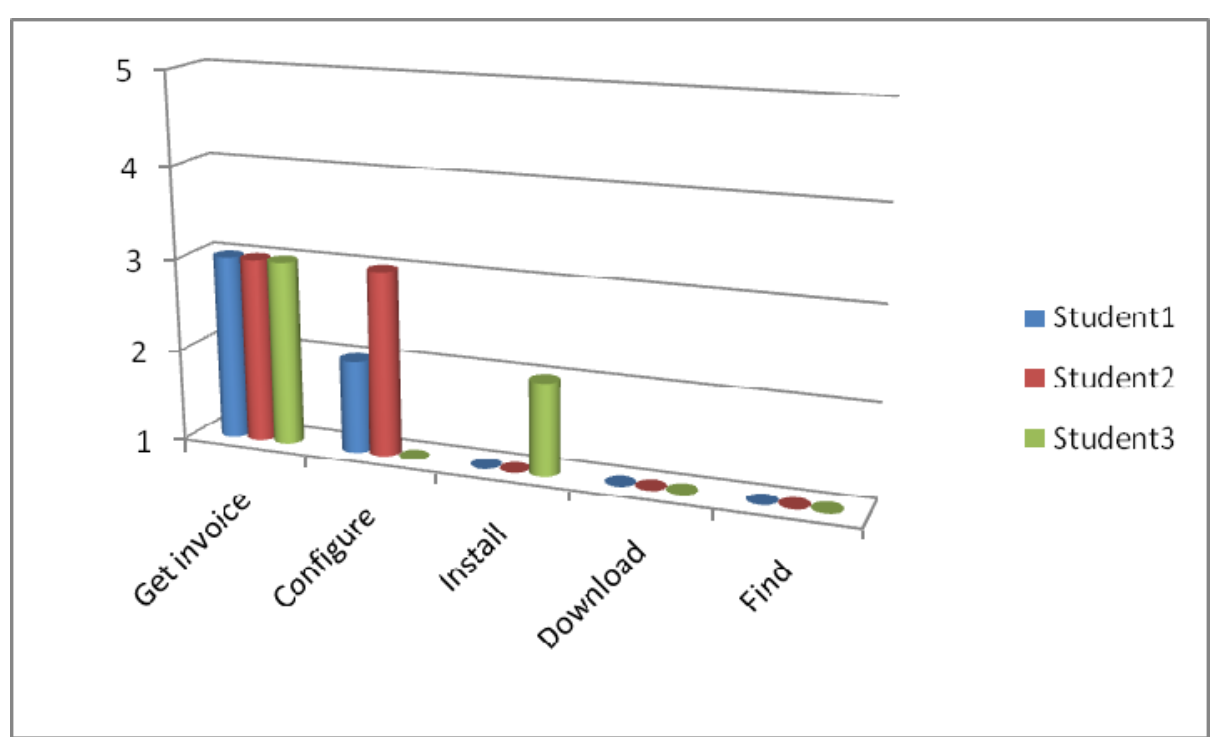

Fig. 2. Perceived ease of use on a likert scale 1-5 (1 very easy) (5 very difficult) 
The students mentioned that in order to accomplish the tasks of finding, downloading, installing the open source ERP not much knowledge is needed. Although, they believe that more knowledge is required in learning the system.

Generally, the deployment process of the open source ERP was assessed as easy. It can be claimed that there are multiple reasons for that. First of all, students mentioned that finding an open source ERP was very easy, as they could Google and pick the most relevant results. The key to finding relevant results were queering the right keywords. When it comes to ERP selection, students mentioned that it was very easy as well. However, most of them judged the quality of the software by Google results. None of the participants has thoroughly assessed the advantages and disadvantages of one product comparing to others, nor did they evaluate the product's technical requirements. Such decisions could affect the overall performance of any open source ERP, if the minimum technical requirements are not met.

Students mentioned throughout the experiment that the software had a very "handy" and good user interface, comparing it to regular software products like Microsoft etc. They also mentioned that the installation and configuration procedure was intuitive and guiding. In other words, they could get clear instructions. Generally, these indicate clear signs of good usability practices as suggested by Nielsen [36].

However, the students felt lack of transparence and information during installation. They felt that more knowledge is required to understand all installation and configuration details. Nevertheless, all three participants succeeded with the installation, being able to run the application. That in turn indicates that little knowledge is needed to install and configure the software, and the role of the user becomes as assisting the process or monitoring, rather than obliged to decide.

The first difficulties came when literally working with the ERP user interface. After having familiarized themselves with available menus, two of the students proceeded immediately with the task using the trial-and-error method. Only one of the students tried to get better informed about the available functions and workflows. That is why the former two had consumed more time and committed more errors while executing the task. The latter used information available on Internet, particularly information on the vendor's site.

Consequently, the appropriate use of software documentation and tutorials might have helped students perform their task faster and with higher qualities. That also supports the idea that users with appropriate training find systems easier and relevant to their job performance. However, Nielsen [36] supports the idea that easy to use system should support learnability, i.e. accomplishing task easily at first encounter with the application.

On one hand, the problem might lie not specifically in the interface design, but rather in the complexity of the ERP software systems. The students confessed that for better usage more knowledge and training is required. On the other hand, the poor performance of two students in terms of time and errors might be also explained by the rush of trying out the system.

Nevertheless, all three students have succeeded to place a sales order, ship and print out the invoice. The resulted invoices do miss important parts of data, and that suggests that they partially neglected the assignment. The problems appeared at 
creating a customized user for the company, i.e. register the company profile, and adding a product to the sales order. In one case, the students blamed the interface of the software, as they could not track the changes to the system.

These problems resulted due to lack of training and proper education of the users. As Bueno and Salmeron [37] mention training and education are necessary before, during and after the system implementation. Consequently, the last task of getting the first invoice was rather hard even for our participants, despite their large experience with other software and high levels of computer skills. Thus, relevant amount of knowledge of the ERP system is a key to perform better job. In terms of time, the deployment assignment was completed in less than one hour.

\subsection{Perceived Usefulness}

The experiment participants perceived the open source ERP as useful. All of the students believed that the software would enrich and help perform the job tasks, as well as reduce useless paperwork.

However, students believed that in order to get better output quality, one should get to learn the system first. The beliefs about result demonstrability were shared. Some of the students believed that at the moment the results are very limited because of the amount of work done. However, they were satisfied to see tangible results in the form of the invoice.

Regarding the image, one of the students mentioned that adopting an open source ERP is becoming a necessity, as well as affect the status and the image of the company in general.

Generally speaking, the perceived usefulness of the open source ERP has been received as positive. That is why two of the students stated that if they were to choose freely, then they would certainly use such software in their company. The left respondent hesitated to give an answer, explaining that it depends on the company and its activity.

In general, perceived usefulness of the ERP system tested was evaluated as positive. In other words, the open source ERP system has delivered the expected benefits, and all of the students find it advantageous for any small company.

All students found the ERP system as beneficial to their job performance, even though they have encountered it for the first time. One of them mentioned that this particular ERP brings more benefits than just rather using Excel or doing paperwork. Of course, it is hard to assess the benefit of a system in such a short time. Rather usefulness is a variable of time, and needs a longer period of time. The same idea is expressed by many scientists in the domain, who claim that majority of company cannot realize the benefits in the first period of time.

The image, voluntariness, result demonstrability factors have also affected positively the perceived usefulness. This can be explained by the background of the students who are all studying information systems. Thus, their general knowledge about ERP and their benefits might have affected their perceived usefulness of the product. However, the students claimed that in comparison to the effort, they could see clear results in the end. 
The resulted invoices are a clear indicator of what can be achieved in shorter than one hour with an open source ERP. Although, the invoices lacked much of the important data, they indicate that with a certain amount of knowledge and training more benefits to the company and overall performance can be gained.

\section{Conclusions}

The research has shown that in order to deploy an open source ERP a relatively short amount of effort - time and knowledge - is needed. The perceived ease of use of the process has been evaluated as relatively easy, with main difficulties appearing in interacting with ERP workflow due to lack of training and little amount of knowledge. However, if appropriate user training and education is applied, greater job performance and output quality can be achieved.

The study has also shown that the output results of the open source ERP are perceived as beneficial to the company and its activity. The students evaluated the perceived usefulness as mostly positive. The factors of job performance, output quality, result demonstrability, voluntariness and image have positively affected the perceived usefulness of the open source ERP system.

\section{References}

1. Verville, J.J., et al.: ERP Acquisition Planning: A Critical Dimension for Making the Right Choice. Long Range Planning 40(1) (2007)

2. Daneva, M.: Understanding Success and Failure Profiles of ERP Requirements Engineering: an Empirical Study. In: 33rd EUROMICRO Conference on Software Engineering and Advanced Applications, EUROMICRO 2007 (2007)

3. The Standish Group, CHAOS Report 1995 (1995)

4. Rapp, J.: Ökat intresse för Open Source. Logica (2009)

5. Johansson, B., Sudzina, F.: ERP systems and open source: an initial review and some implications for SMEs. Journal of Enterprise Information Management 21(6), 649 (2008)

6. Morabito, V., Pace, S., Previtali, P.: ERP Marketing and Italian SMEs. European Management Journal 23(5), 590-598 (2005)

7. Xu, L., Brinkkemper, S.: Concepts of product software. European Journal of Information Systems 16(5), 531-541 (2007)

8. Lengnick-Hall, C.A., Lengnick-Hall, M.L., Abdinnour-Helm, S.: The role of social and intellectual capital in achieving competitive advantage through enterprise resource planning (ERP) systems. Journal of Engineering and Technology Management 21(4), 307 330 (2004)

9. Rolland, C., Prakash, N.: Bridging the Gap Between Organisational Needs and ERP Functionality. Requirements Engineering 5(3), 180-193 (2000)

10. Kumar, K., Van Hillegersberg, J.: ERP experiences and evolution. Communications of the ACM 43(4), 22-26 (2000)

11. Wier, B., Hunton, J., HassabElnaby, H.R.: Enterprise resource planning systems and nonfinancial performance incentives: The joint impact on corporate performance. International Journal of Accounting Information Systems 8(3), 165-190 (2007) 
12. Koch, C.: ERP-systemer: erfaringer, ressourcer, forandringer, p. 224. Ingeniøren-bøger, København (2001)

13. Millman, G.J.: What did you get from ERP, and what can you get? Financial Executives International 5, 15-24 (2004)

14. Hammer, M., Champy, J.: Reengineering the corporation: a manifesto for business revolution, 1st edn., p. 223. Harper Business, New York (1993)

15. Davenport, T.: Mission Critical: Realizing the Promise of Enterprise Systems. Harvard Business School Press, Boston (2000)

16. Larsen, M., Myers, M.D.: When Success Turns into Failure: A Package-Driven Business Process Re-engineering project in the Financial Services Industry. Journal of Strategic Information Systems 8, 395-417 (1998)

17. Scott, J.E., Vessey, I.: Implementing Enterprise Resource Planning Systems: The Role of Learning from Failure. Information Systems Frontiers 2(2), 213 (2000)

18. Robey, D., Ross, J.W., Boudreau, M.-C.: Learning to Implement Enterprise Systems: An Exploratory Study of the Dialectics of Change. Journal of Management Information Systems 19(1), 17-46 (2002)

19. Hedman, J., Borell, A.: ERP systems impact on organizations. In: Grant, G. (ed.) ERP \& Data Warehousing in Organizations: Issues and Challenges, pp. 1-21. Idea Group Publishing, Hershey (2003)

20. Davenport, T.: Holistic management of mega-package change: The case of SAP. Center of Business Innovation, Ernest \& Young LLP, Boston (1996)

21. Johansson, B., Newman, M.: Competitive advantage in the ERP system's value-chain and its influence on future development. Enterprise Information Systems 4(1), 79-93 (2010)

22. Wieder, B., et al.: The impact of ERP systems on firm and business process performance. Journal of Enterprise Information Management 19(1), 13-29 (2006)

23. Jacobson, S., et al.: The ERP Market Sizing Report, 2006-2011. AMR Research (2007)

24. Parr, A.N.: A Taxonomy of ERP Implementation Approaches (2000)

25. Shehab, E.M., et al.: Enterprise resource planning: An integrative review. Business Process Management Journal 10(4), 359-386 (2004)

26. Fougatsaro, V.G.: A study of open source ERP systems. School of Management. Blekinge Institute of Technology (2009)

27. Carvalho, R.A.: Issues on evaluating Free/open source ERP systems. In: Research and Practical Issues of Enterprise Informations Systems, pp. 667-676. Springer Verlag New York Inc., New York (2007)

28. Bajaj, A.: ERP for SMEs (2008),

http://knol.google.com/k/erp-for-sme-s\#

29. Sammon, D., Adam, F.: Justifying an ERP investment with the promise of realising business benefits. In: 15th European Conference on Information Systems, pp. 1655-1668. University of St. Gallen (2007)

30. European Commission, SME Definition: Recommendation 2003/361/EC Regarding the SME Definition (2003)

31. Laukkanen, S., Sarpola, S., Hallikainen, P.: Enterprise size matters: objectives and constraints of ERP adoption. Journal of Enterprise Information Management 20(3), 319-334 (2007)

32. De Carvalho, R.A., Johansson, B.: ERP Licensing Perspectives on Adoption of ERPs in Small and Medium-sized Enterprises. In: Chaudbry, S. (ed.) The IV IFIP International Conference on Research and Pratical Issues of Enterprise Systems (CONFENIS), Rio Grande do Norte, Barcellos de Andrade, Inez (2010) 
33. Suraweera, T., Remus, U., Wakerley, S.: Dynamics of Knowledge Leverage in ERP Implementation. In: ACIS 2007 Proceedings. Paper 58 (2007)

34. Venkatesh, V., Davis, F.D.: A theoretical extension of the technology acceptance model: Four longitudinal Field Studies. Management Science 46(2), 186-204 (2000)

35. Davis, F.D.: Perceived usefulness, perceived ease of use, and user acceptance of information technology. MIS Quarterly 13(3), 319-340 (1989)

36. Nielsen, J.: Usability 101: Introduction to Usability (2011), http://www. useit.com/alertbox/20030825.html (cited May 1, 2011)

37. Bueno, S., Salmeron, J.L.: TAM-based success modeling in ERP. Journal Interacting with Computers 20(6), 515-523 (2008) 